Ann. Sci. forest., 1981, 38 (2), 283-298

\title{
Détermination du module d'Young du bois par une méthode dynamique sur carottes de sondage
}

\author{
Voichita BUCUR \\ avec la collaboration technique de A. PERrin \\ Station de Recherches sur la Qualité des Bois, \\ Centre de Recherches forestières de Nancy, I.N.R.A., \\ Champenoux, 54280 Seichamps
}

\begin{abstract}
Résumé
La méthode de la vitesse de propagation d'ondes ultrasonores a été utilisée pour estimer le module d'Young selon l'axe longitudinal sur des carottes de sondage de $5 \mathrm{~mm}$ de diamètre.

Les résultats ont été comparés avec la méthode normalisée basée sur la mesure du module d'Young en flexion statique sur éprouvettes de $2 \times 2 \times 30 \mathrm{~cm}$, et du module de rupture.

Les coefficients de corrélation très élevés obtenus font apparaître des liaisons extrêmement étroites entre les trois mesures.

Ainsi, la méthode à ultrasons sur carottes est capable destimer les constantes élastodynamiques du bois.

Des futures recherches sont nécessaires pour expliquer l'influence de la petite taille de la carotte sur la mesure.
\end{abstract}

\section{Introduction}

Les carottes de sondage de $5 \mathrm{~mm}$ ont été utilisées à l'origine pour obtenir des informations sur l'âge et l'accroissement annuel des arbres.

Depuis environ 25 ans, elles servent également à l'appréciation non destructive de la qualité du bois (hétérogénéité, valeur papetière, contrainte de croissance) (Pol.ge, 1980).

En ce qui concerne les propriétés mécaniques, seule la détermination de la dureté du bois a fait lobjet des travaux de Tryon et al. (1976).

L'étude sur carottes de sondage des autres propriétés mécaniques du bois n’avait pas été abordéc jusqu'à présent à cause sans doute de la taille très faible de l'échantillon. D’autre part, la détermination des caractéristiques mécaniques d'un bois par les méthodes classiques normalisées nécessite une grande quantité de matériel ligneux. Elle n'est réalisable qu’à partir d'échantillons importants entraînant l’abattage de laarbre. 
Mais, ces méthodes, tout en fournissant des résultats rigoureux, exigent une somme de travail considérable pour le prélèvement et la préparation des échantillons, et présentent l'inconvénient d'être peu économiques.

On a dès lors cherché à utiliser des méthodes non destructives pour déterminer les propriétés mécaniques du bois sur carottes, et notamment des méthodes acoustiques à ultrasons car la mesure, facile et rapide, de la vitesse des ondes ultrasonores permet d'obtenir, par le calcul, les constantes élastiques du bois.

Cette méthode a fait l'objet de quelques études sur bois massif (LEE, 1958 ; Burmester, 1965 ; Facaoaru \& Bucur, 1974) et sur le bois utilisé comme matériau de construction sous forme de poutres en lamellé-collé (Elvery, Nwokoye, 1970), ou pour l'appréciation objective du bois de résonance (BucuR, 1980).

Des travaux récents sur les panneaux de particules (Dunlop, 1980) montrent la validité de la méthode pour le contrôle industriel en continu.

Parmi les constantes élastiques, les modules d'Young ont suscité un intérêt tout à fait particulier car ils peuvent être calculés à partir de la vitesse de propagation des ondes ultrasonores et de la densité du matériau.

Le besoin de pouvoir mesurer de façon non destructive les propriétés mécaniques du bois nous a encouragée à tenter d'appliquer la méthode de la vitesse de propagation des ondes ultrasonores à de simples carottes de sondage brutes de $5 \mathrm{~mm}$ de diamètre.

L'objet de cette note est de comparer les résultats obtenus par la méthode classique statique, sur éprouvettes normalisées (module d'élasticité et module de rupture) d'une part, et par la méthode dynamique (module d'élasticité) à ultrasons sur carottes de sondage d'autre part.

\section{1. - Considérations théoriques sur la méthode}

\section{1. - Définition des constantes élastiques}

Le bois, solide anisotrope et non homogène, obéit à la loi de Hooke généralisée :

$$
\text { où } \begin{aligned}
\sigma_{\mathrm{i}} & =\mathrm{C}_{\mathrm{ij}} \cdot \varepsilon_{\mathrm{j}} \\
\varepsilon_{\mathrm{i}} & =\mathrm{S}_{\mathrm{ij}} \cdot \sigma_{\mathrm{j}}
\end{aligned}
$$

où

$$
\begin{aligned}
\sigma_{\mathrm{i}} & \text { est la matrice des contraintes } \\
\mathrm{C}_{\mathrm{ij}} & \text { est la matrice des rigidités } \\
\varepsilon_{\mathrm{i}} & \text { est la matrice des déformations } \\
\mathrm{S}_{\mathrm{ij}} & \text { est la matrice des souplesses }
\end{aligned}
$$

Du fait de la symétrie élastique suivant les trois axes : longitudinal, radial et tangentiel, par rapport à la direction de croissance de l'arbre, le nombre de constantes $S_{i j}$ pour le bois, considéré comme un système orthorhombique, est de 9 . 
Ainsi .

$$
\left(\begin{array}{c}
\varepsilon_{1} \\
\varepsilon_{2} \\
\varepsilon_{3} \\
\gamma_{4} \\
\gamma_{5} \\
\gamma_{6}
\end{array}\right)=\left(\begin{array}{cccccc}
S_{11} & S_{12} & S_{13} & 0 & 0 & 0 \\
S_{21} & S_{22} & S_{23} & 0 & 0 & 0 \\
S_{31} & S_{32} & S_{33} & 0 & 0 & 0 \\
0 & 0 & 0 & S_{14} & 0 & 0 \\
0 & 0 & 0 & 0 & S_{55} & 0 \\
0 & 0 & 0 & 0 & 0 & S_{66}
\end{array}\right)-\left(\begin{array}{c}
\sigma_{1} \\
\sigma_{2} \\
\sigma_{3} \\
\tau_{1} \\
\tau_{5} \\
\tau_{6}
\end{array}\right)
$$

Les six constantes élastiques, trois modules d'Young et trois modules Coulomb sobtiennent par identification avec $1 / S_{11}, 1 / S_{2: 2}, 1 / S_{33}$ et $1 / S_{14}, 1 / S_{55.5}, 1 / S_{66}$.

D'autre part, entre les modules d'Young et les coefficients Poisson, on a les relations suivantes :

$$
\frac{v_{12}}{\mathrm{E}_{1}}=\frac{v_{21}}{\mathrm{E}_{2}} ; \frac{v_{23}}{\mathrm{E}_{22}}=\frac{v_{32}}{\mathrm{E}_{3}} ; \frac{v_{31}}{\mathrm{E}_{31}}=\frac{v_{13}}{\mathrm{E}_{1}}
$$

\section{2. - Mesures ultrasonores de constantes élastiques}

On démontre que, pour un solide orthotrope (VINH, 1979), on a :

$$
\begin{aligned}
& \mathrm{c}_{\mathrm{ii}}-\omega^{2} \mathrm{~V}_{\mathscr{B}}=0 \\
& c_{i j}-g V_{\tau}^{2}=0
\end{aligned}
$$

La première équation correspond à une onde longitudinale de vitesse $\mathrm{V}_{\mathscr{L}}$ (pour laquelle la polarisation de l'onde est confondue avec la direction de propagation), telle que :

$$
\mathrm{V}_{\mathscr{E}}=\sqrt{\frac{\overline{\mathrm{c}_{\mathrm{i}}}}{\rho}} \text { où } \mathrm{c}_{\mathrm{ii}}=\mathrm{V}_{\mathscr{E}}^{2} \cdot 0
$$

La deuxième équation correspond à une onde transversale, de vitesse $V_{\tau}$ (la polarisation de l'onde est perpendiculaire à la direction de propagation) :

$$
\mathrm{V}_{\tau}=\sqrt{\frac{\overline{\mathrm{c}_{\mathrm{ij}}}}{\underline{\underline{e}}}} \text { où } \mathrm{c}_{\mathrm{ii}}=\mathrm{V}_{\tau}^{2} \cdot \underline{\mathrm{g}}
$$

\section{2. - Matériel et méthode}

\section{1. - Echantillonnage}

18 carottes de $5 \mathrm{~mm}$ et 18 éprouvettes normalisées ont été prélevées dans 9 Hêtres (Fagus silvatica L.) provenant de la forêt d'Epinal. 
Le schéma de débit des éprouvettes normalisées à proximité immédiate des carottes est représenté en figure 1 .
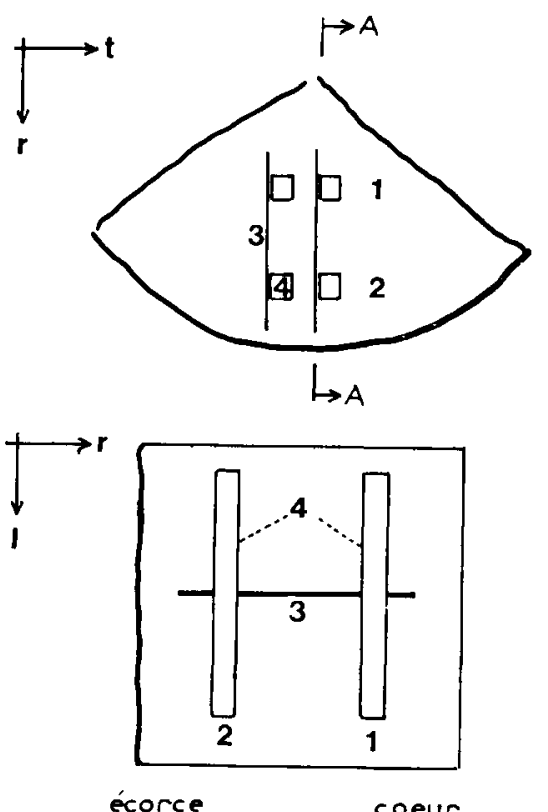

section A A

1 zonecôlé coeur; 2 zone collé écorce

3 corolte; 4 eprouvelle

Figure 1

Schéma de débit des éprouvettes

Specimen selection procedure

Dans chaque arbre, on a prélevé deux carottes radiales de $5 \mathrm{~mm}$ de diamètre.

Sur chacune, on a délimité deux zones, une du côté écorce, et la deuxième voisine du milieu de l'arbre, zone dénommée arbitrairement zone côté cœur, simplement pour la différencier de la zone côté écorce.

Des éprouvettes normalisées d'orientation longitudinale de $2 \times 2 \times 30 \mathrm{~cm}$ ont été prélevées au voisinage immédiat de chaque zone pour chaque carotte de façon telle que les milieux des zones de carottes et des éprouvettes coïncident. Cette précaution a été prise en vue d'éliminer les effets de la variabilité du bois, aussi bien dans le sens longitudinal que dans le sens radial. 
Il convient de mentionner que les deux carottes ne pouvant être à la fois parallèles et exactement radiales, la priorité a été donnée au parallélisme, de façon à ne pas trop éloigner éprouvettes et carottes de la zone écorce.

\section{2. - Méthode de mesure des modules d'Young}

\subsection{1. - Sur éprouvettes normalisées}

\subsection{1. - Essais statiques}

Les essais statiques ont été effectués selon la norme française NF 51-002; chaque éprouvette a été essayée en flexion statique, sur une machine TESTWELL de 5 tonnes, avec l'effort exercé tangentiellement aux cernes, la distance entre appuis est de $28 \mathrm{~cm}$, la charge appliquée au milieu de la face radiale de l'éprouvette par un couteau cylindrique de $3 \mathrm{~cm}$ de diamètre, de façon que sa flèche augmente d'environ $5 \mathrm{~mm} / \mathrm{mn}$.

La déformation a été mesurée au milieu de l'éprouvette, avec un comparateur de précision de $0,01 \mathrm{~mm}$, et la force d'appui avec une précision de $1 \mathrm{~kg}$.

On a effectué trois séries de mesures pour chaque éprouvette, avec charges successives de 10,20 et $30 \mathrm{~kg}$.

La linéarité de la déformation a été contrôlée aussi sur l'enregistrement graphique en coordonnées force-déformation.

A la fin de la série de mesure dans le domaine élastique, la sollicitation a été conduite jusqu'à la rupture.

Le module d'Young et le module de rupture ont été calculés selon les relations simples de résistance des matériaux pour une poutre sollicitée à la flexion.

\subsection{2. - Essais dynamiques}

En ce qui concerne les essais dynamiques sur les éprouvettes normalisées, on a utilisé un ausculteur dynamique AU 80 à ultrasons, de fréquence $80 \mathrm{KH}_{\mathrm{Z}}$.

La relation de calcul pour le module dynamique brut (car on ne prend pas en compte la correction due au coefficient Poisson) est la suivante :

$$
\mathrm{E}_{\mathrm{dynamique}}=\mathrm{v}^{* 2} \cdot \mathrm{g}
$$

dont 0 est la masse volumique en $\mathrm{kg} / \mathrm{m}^{3}$ et $\mathrm{V}$ la vitesse de propagation d'ultrasons en $\mathrm{m} / \mathrm{s}$, calculée comme le rapport entre la longueur de l'éprouvette et le temps lu sur l'appareil.

Par la suite, dans un but de simplification, on assimilera le module d'Young dynamique à la valeur du module brut mentionné plus haut.

La mesure de la vitesse a été effectuée selon les trois axes d'anisotropie du bois, mais le présent compte-rendu fait référence seulement à la direction longitudinale, qui est d'ailleurs la seule pour laquelle une comparaison puisse être faite avec l'éprouvette normalisée. 

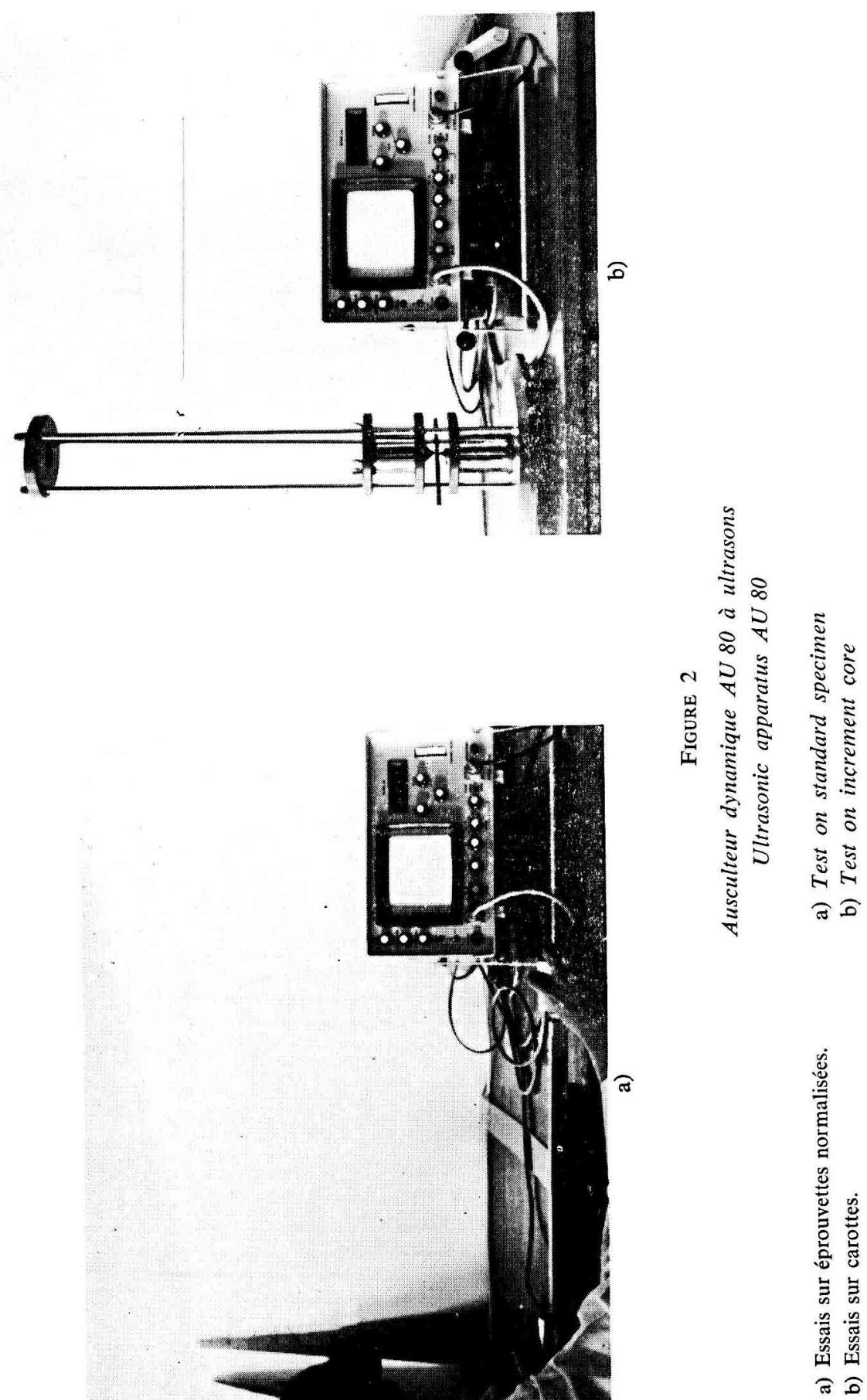


\subsection{2. - Sur carottes de sondage}

Les carottes de sondage ont été étudiées seulement en régime dynamique avec le même appareil que les éprouvettes normalisées.

La mesure de la vitesse de propagation dans la direction longitudinale a été faite, comme dans la figure $2 b$, avec trois répétitions sur le même point pour chaque carotte, côté écorce et côté cœur. Le nombre de mesures effectuées a donc été :

- pour les essais statiques, module d'Young, sur éprouvettes normalisées, de : 9 arbres $\times 4$ éprouvettes $\times 3$ répétitions $=108$

- pour les essais de rupture:

$$
9 \text { arbres } \times 4 \text { éprouvettes }=36
$$

- pour les essais dynamiques, sur éprouvettes normalisées, de :

$$
9 \text { arbres } \times 4 \text { éprouvettes } \times 3 \text { répétitions }=108
$$

- pour les carottes :

9 arbres $\times 2$ carottes $\times 2$ zones de mesure $\times 3$ répétitions $=108$

\section{3. - Résultats et discussions}

Les résultats sont présentés dans le tableau 1.

Les modules dynamiques, statiques et de rupture du côté cœur sont significativement supérieurs à ceux du côté écorce, traduisant une baisse des propriétés mécaniques avec l'âge, en liaison probable avec une diminution des largeurs de cernes. Les valeurs mesurées sur éprouvettes normalisées en régime dynamique sont les plus élevées; cette différence est due à la nature de la sollicitation, car on sait que l'essai statique induit dans l'éprouvette des changements des propriétés rhéologiques, tandis que l'essai dynamique ne détermine pas des modifications de structure.

\section{Tableau 2}

Comparaison entre les modules dynamiques et statiques mesurés sur carottes et sur ćprouvettes normalisées

Comparison between dynamical and statical modulus of elasticity on increment cores

\begin{tabular}{|c|c|c|c|}
\hline Comparaison & Variables comparées & Zone & Critére $\mathrm{t}$ \\
\hline 1. - Carotte-éprouvette & $\begin{array}{l}\text { Module dynamique avec } \\
\text { module dynamique } \ldots \ldots \ldots\end{array}$ & $\begin{array}{l}\text { Ecorce } \\
\text { Cocur }\end{array}$ & $\begin{array}{l}4,8113 * * \\
6,3136 * * *\end{array}$ \\
\hline 2. - Eprouvette-éprouvette & $\begin{array}{l}\text { Module dynamique avec } \\
\text { module statique } \ldots \ldots \ldots\end{array}$ & $\begin{array}{l}\text { Ecorce } \\
\text { Coeur }\end{array}$ & $\begin{array}{l}5,2621 * * * \\
7,7937 * * * *\end{array}$ \\
\hline
\end{tabular}
and standard specimens 


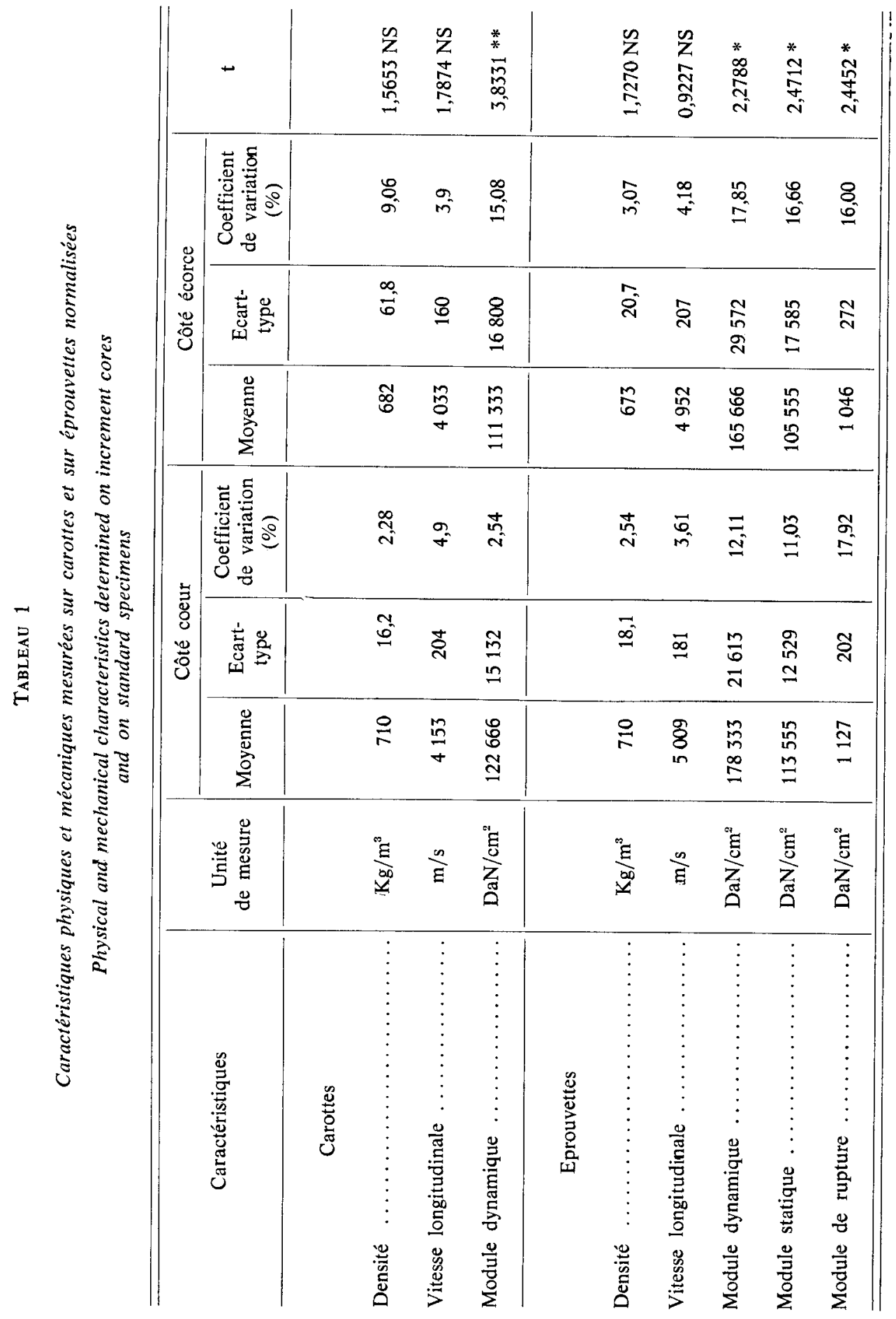


Le tableau 2 montre que les différences entre les modules dynamiques mesurés sur carottes et sur éprouvettes normalisées sont très significatives, mais pour les deux zones, moins marquées que celles qui existent entre module statique et module dynamique mesurés sur le même matériel (éprouvettes normalisées).

Si on se reporte à la même densité de $710 \mathrm{~kg} / \mathrm{m}^{3}$, mentionnée dans le premier tableau, le rapport entre les modules dynamiques sur éprouvette et sur carotte est voisin de 1,5.

L'écart entre les deux vitesses $(856 \mathrm{~m} / \mathrm{s})$, qui est à l'origine de cette différence, est dû probablement à l'interaction entre la géométrie de la carotte et la longueur d'onde. Des recherches sont en cours à ce sujet.

Bien que les valeurs absolues du module mesurées sur carottes et sur éprouvettes ne concordent pas, l'intérêt de la méthode ultrasonique demeure, car elle permet de mettre en évidence de façon rapide et simple les différences relatives de la qualité du bois entre individus ou groupe d'individus en fonction du milieu, de la sylviculture ou de l'hérédité.

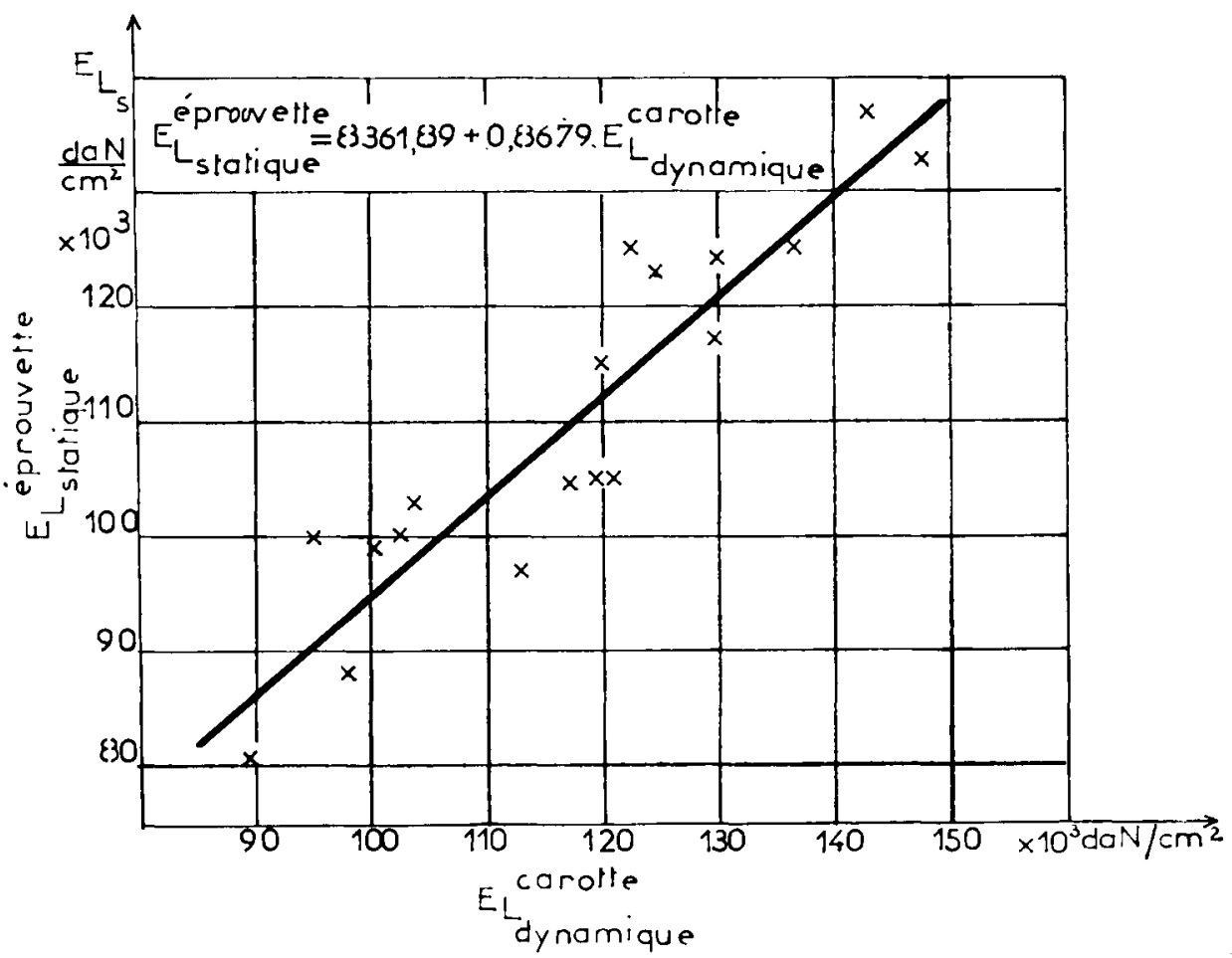

Figure 3

Variation du module statique sur éprouvette normalisée en fonction du module dynamique sur carotte

Relationship between dynamical Young's modulus on standard specimen and dynamical Young's modulus on increment core 


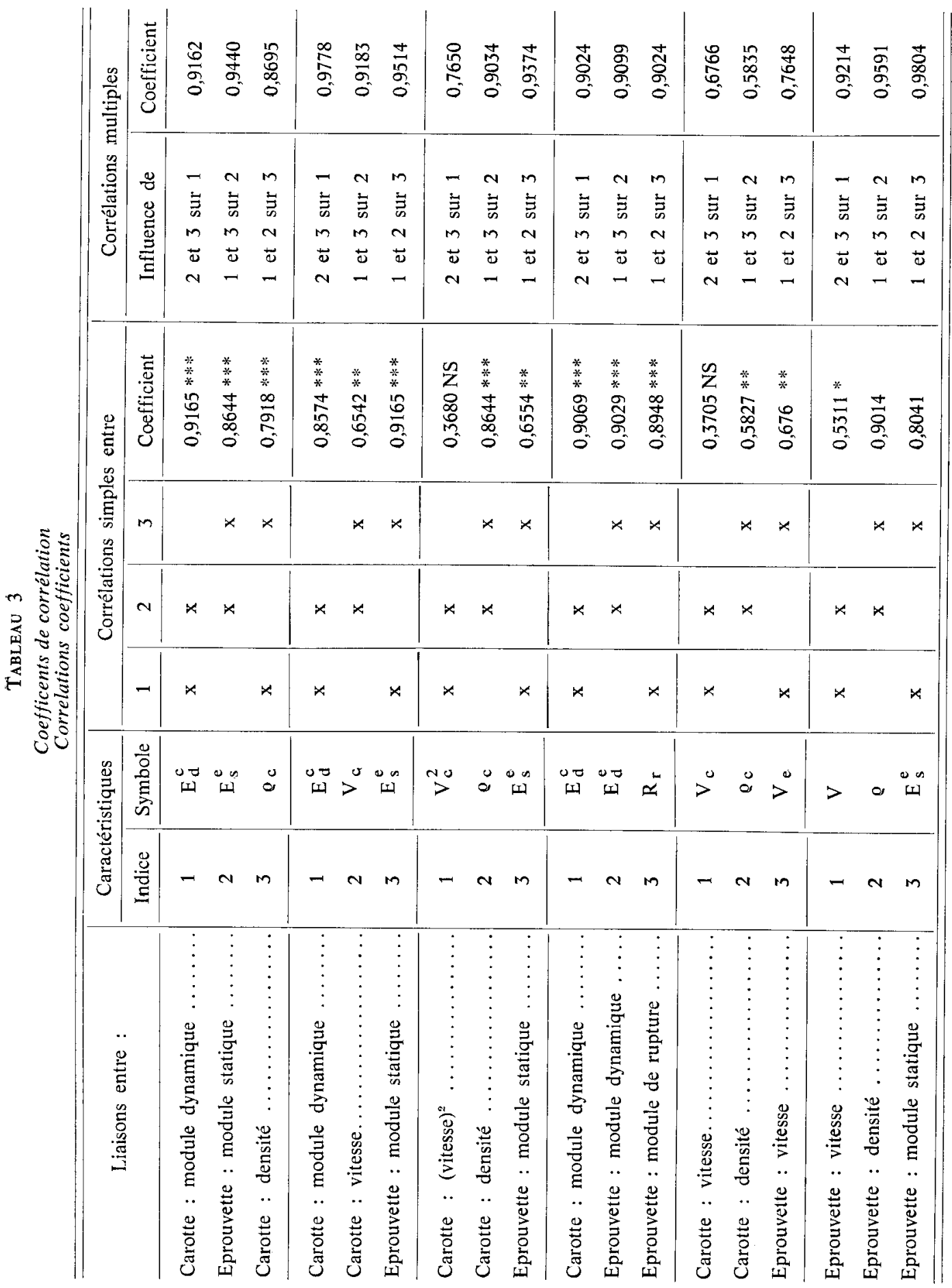


L'intensité de la liaison entre les caractéristiques mesurées sur carottes et sur éprouvettes a été cherchée à partir du coefficient de corrélation simple (tableau 3).

Le coefficient le plus intéressant est celui qui lie le module dynamique sur carotte au module statique sur éprouvette normalisée (figure 3); dans le cas étudié, il est de $0,9165 * * *$.

D'autre part, si on prend en compte la corrélation entre le module dynamique sur carotte et le module dynamique sur éprouvette normalisée (figure 4), on obtient un coefficient de corrélation de $0,9065 * * *$.

Une autre valeur à mentionner est la valeur du coefficient de corrélation entre le module dynamique de la carotte et le module de rupture de l'éprouvette (figure 5) égal à $0,8948 * * *$.

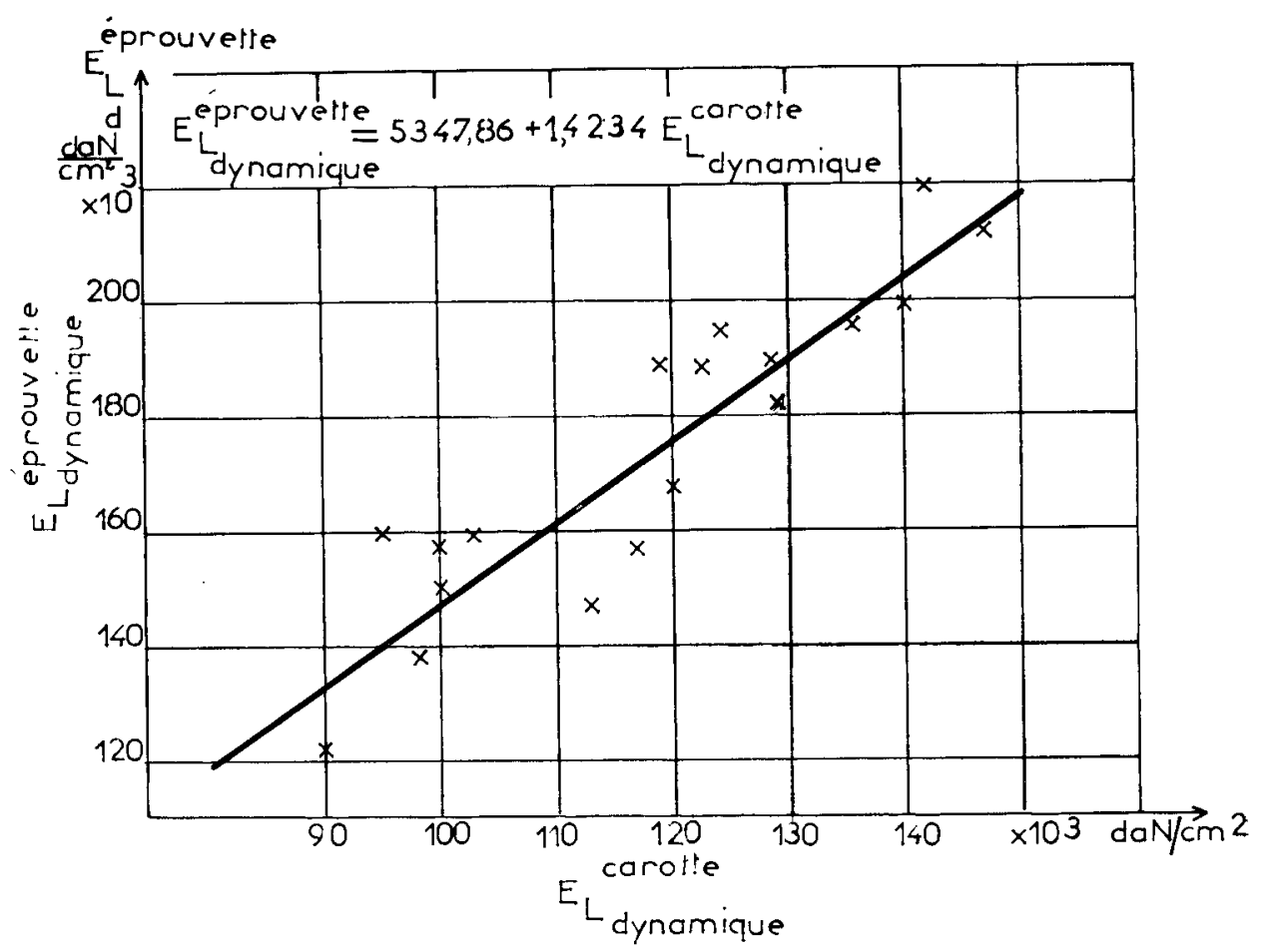

FIGURE 4

Variation du module dynamique sur éprouvette normalisée en fonction du module dynamique sur carotte

Relationship between statical Young's modulus on standard specimen and on increment core 


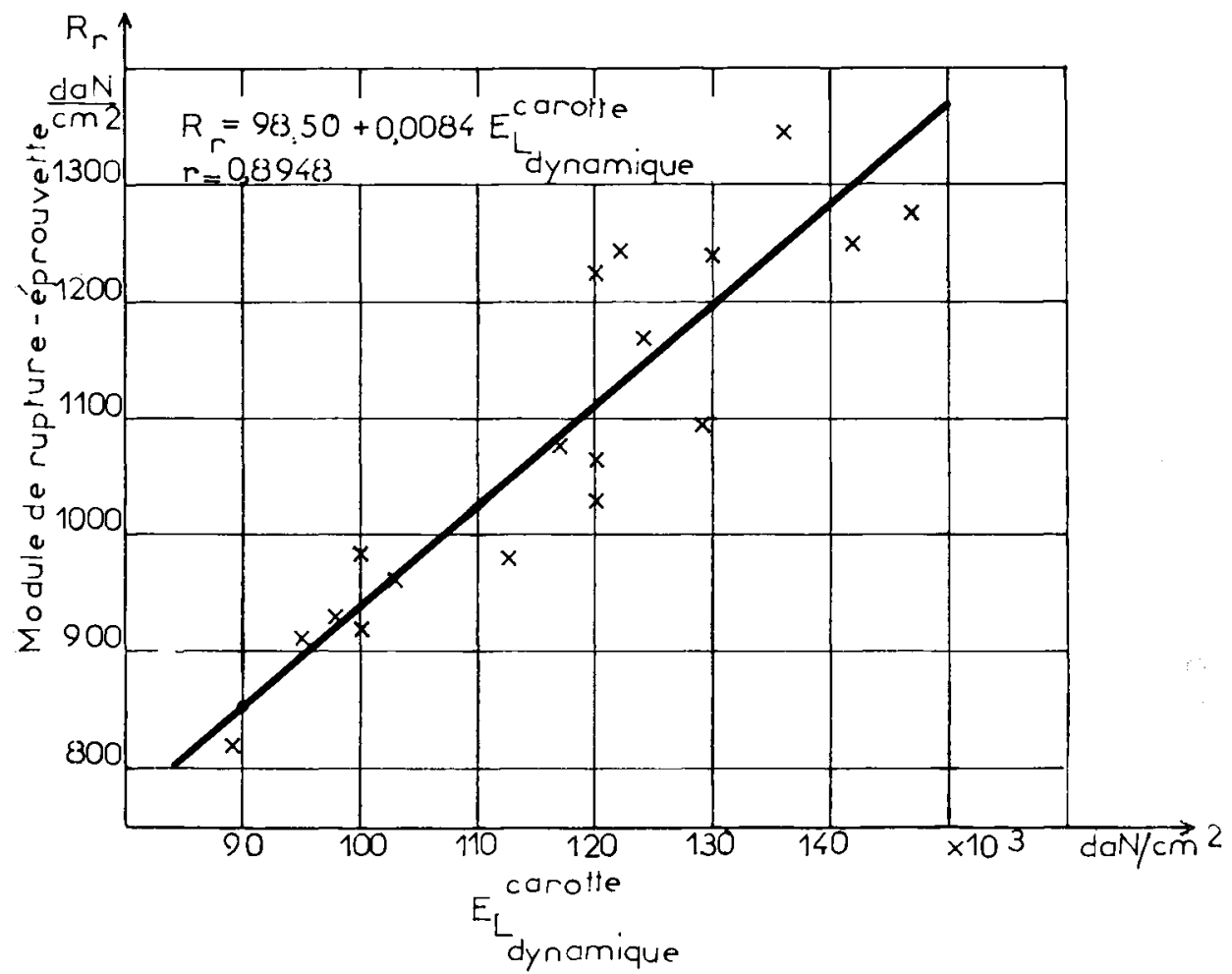

FIGURE 5

Variation de la résistance à la flexion statique en fonction du module dynamique sur carotte

Relationship between modulus of rupture in bending on standard specimen and Young's modulus on increment core

Les équations de régression linéaire du module statique sur éprouvette en fonction de plusieurs variables explicatives mesurées sur carotte ou sur éprouvette sont données dans le tableau 4.

Le pourcentage de la variation du module statique sur éprouvette expliqué par les variables explicatives varie de 42 p. 100 à 96 p. 100.

La variable qui fournit la meilleure explication du module statique est le module dynamique sur éprouvette, ce qui est tout à fait normal, les deux modules étant mesurés sur le même échantillon. En second licu, on trouve le module dynamique sur carotte et le module de rupture sur éprouvette. En troisième place, se trouve la densité de la carotte, et en quatrième, la vitesse sur carotte.

Ainsi, le module dynamique sur carotte, calculé à partir de la vitesse des ultrasons et de la densité du bois, permet d'expliquer le module statique sur éprouvette normalisće, mieux que la densité seule. 


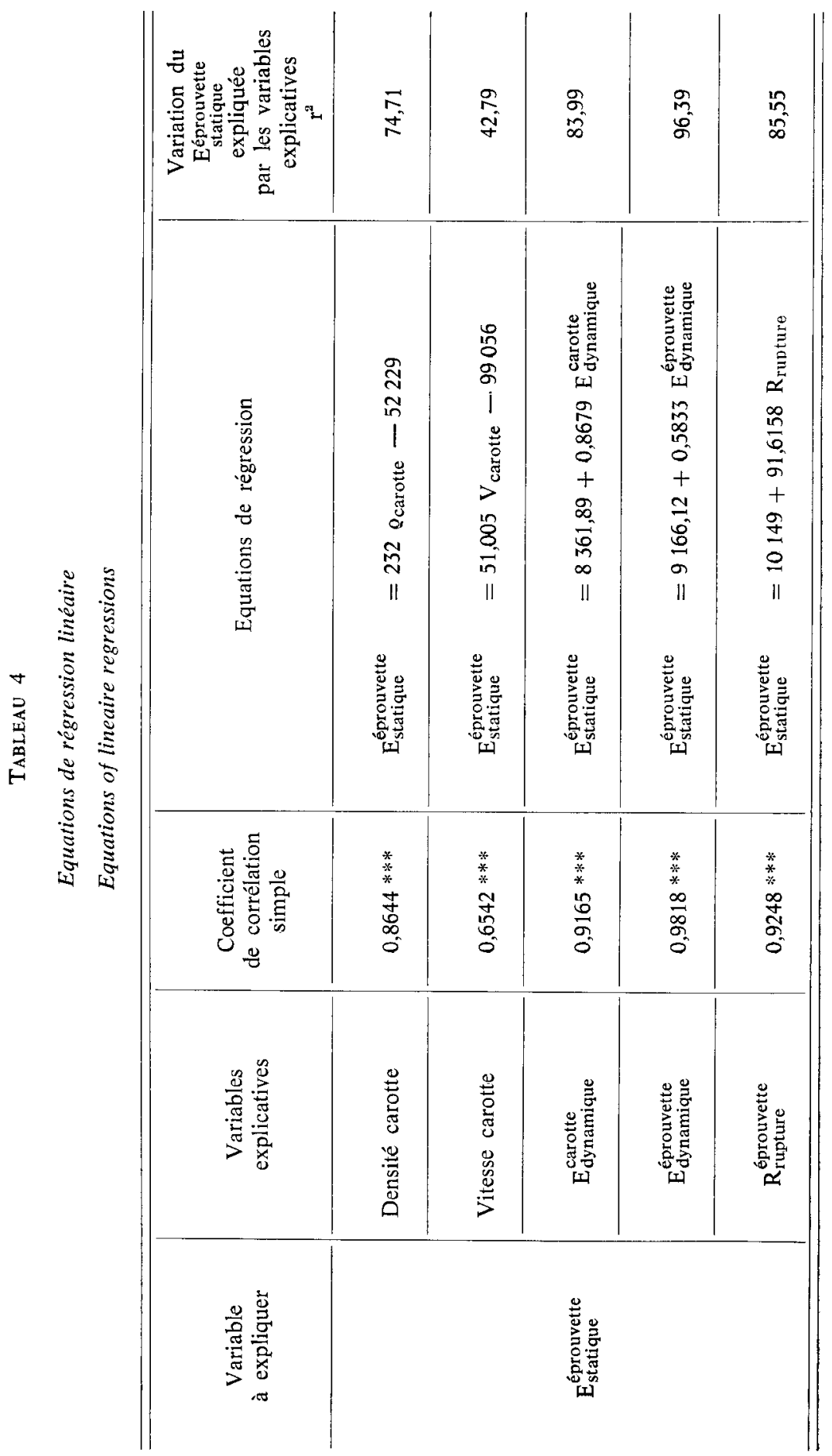




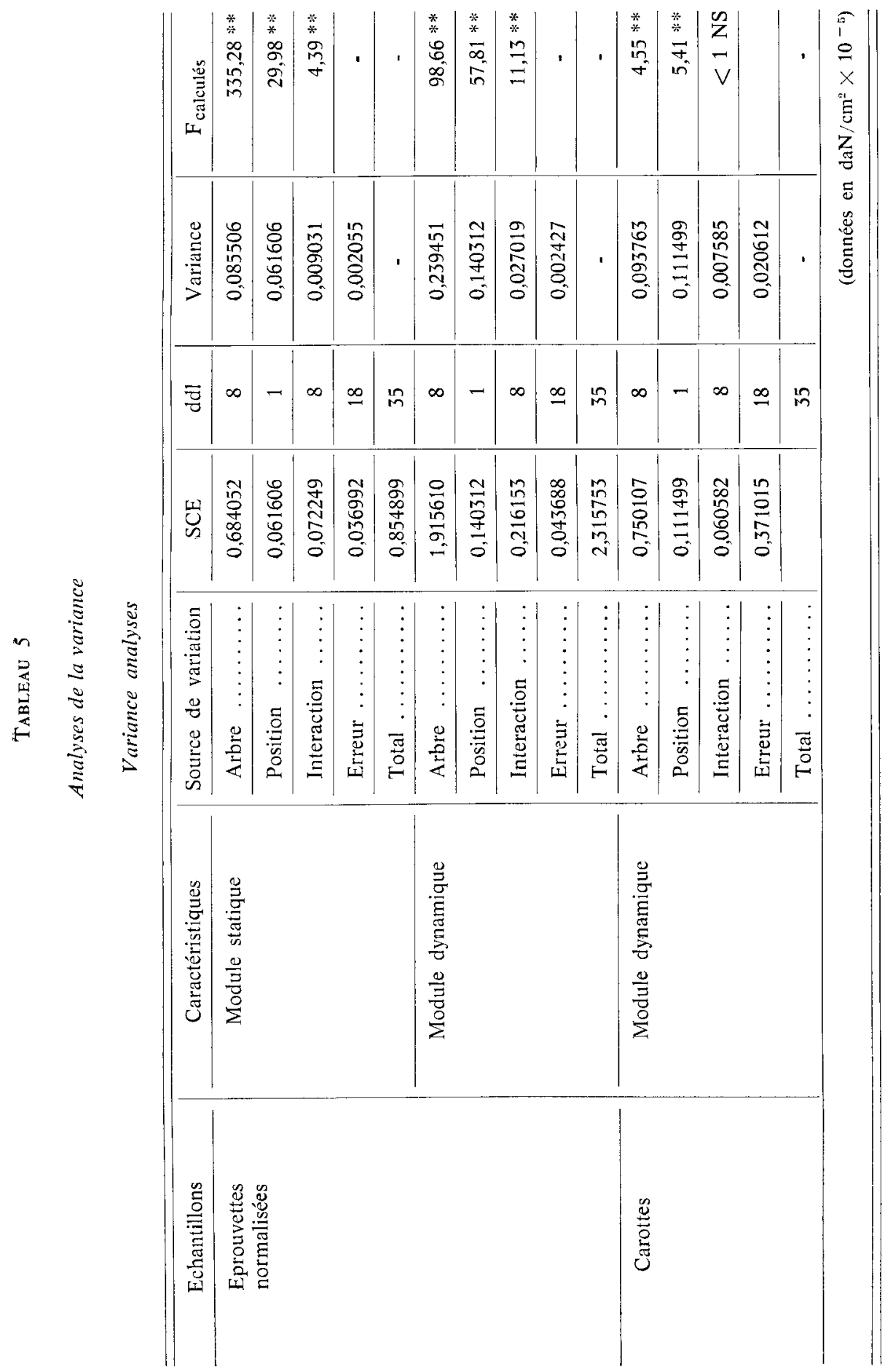


Pour préciser dans quelle mesure le module dynamique sur carotte peut être considéré comme discriminant par rapport au module statique ou dynamique sur éprouvette normalisée, des analyses de variance ont été faites (tableau 5) pour chaque type d'éprouvette, et pour chaque régime de sollicitation.

L'effet arbre et l'effet position de l'échantillon dans l'arbre ont été étudiés. On peut faire les commentaires suivants :

- pour l'éprouvette normalisée, l'effet arbre et l'effet position mis en évidence sont significatifs à 1 p. 100 ; l'erreur est la même pour le module dynamique et pour le module statique ;

- pour la carotte, l'effet arbre est significatif à 1 p. 100, mais l'effet position (différence entre côté écorce et cœur) ne l'est qu'à 5 p. 100 ; l'erreur est presque 10 fois plus grande sur la carotte de sondage que sur l'éprouvette normalisée.

Ces observations pourraient paraître décourageantes, mais il convient de noter qu'en dépit des très faibles dimensions des échantillons $(5 \mathrm{~mm}$ dans le sens longitudinal), la mesure du module dynamique par ultrasons permet de différencier les arbres entre eux, ce qui est le but principal poursuivi.

L'erreur expérimentale qui affecte ces mesures peut être attribuée à deux causes principales:

— sensibilité insuffisante du capteur qui n'a pas été conçu pour des objets d'aussi petite taille, mais qui pourra sans doute être amélioré ;

- difficulté de positionnement des carottes, entraînant des risques de nonparallélisme entre le fil du bois et le train d'ondes ultrasoniques.

\section{Conclusion}

Les résultats obtenus par l'essai statique ou dynamique sur l'éprouvette normalisée et par l'essai dynamique sur carotte font apparaître des liaisons extrêmement étroites entre les trois mesures.

Il est ainsi d'ores et déjà possible d'obtenir par une méthode non destructive une appréciation de la résistance mécanique du bois à partir d'une carotte de sondage. A l'heure actuelle, cette estimation est satisfaisante en valeur relative, c'est-àdire pour des comparaisons entre individus ou populations.

Une étape suivante consistera à déterminer les corrections nécessaires pour avoir des valeurs du module d'élasticité qui soient exactes en valeur absolue sur carottes et comparables à celles du module statique mesuré sur éprouvette normalisée.

Reçu pour publication en janvier 1981. 


\section{Summary}

Wood dynamical Young's modulus determination on increment core

Velocity ultrasonic method was used to estimate the Young's modulus on longitudinal anisotropic axis on $5 \mathrm{~mm}$ diameter increment core.

Results were compared with standard method, based on Young's modulus in static bending $2 \times 2 \times 30 \mathrm{~cm}$ specimen and on rupture modulus.

Very high correlation coefficients obtained put in evidence the high dependence between the 3 mentionned sollicitations.

Therefore the ultrasonic method is capable to estimate the wood elasto-dynamical constants.

Further researches are need to explaine the influence of small dimension of core on the measurement.

\section{Références bibliographiques}

AFNOR B 51-008. Bois, essai de flexion statique.

Burmester A., 1965. Zusammenhang zwischen Schallgeschwindigkeit und morphologischen physikalischen und mechanischen Eigenschaften von Holz. Holz als Roh und Werkstoff, 23 (6), 227-236.

Bucur V., 1980. Modifications des propriétés acoustiques du bois de résonance sous l'effet de sollicitations de longue durée. Ann. Sci. for., 37 (3), 249-264.

Dagnelie P., 1977. Analyse statistique à plusieurs variables. Presses Agronomiques de Gembloux, Belgique.

Dunlop J.I., 1980. Testing of particle board by acoustic technique. Wood Science and Technology, 14 (1), 69-78.

Elvery R., Nwokoye D.N., 1970. Strength assessment of timber for glued laminated beems. Symposium on non-destructive testing of concrete and timber, London, The Institute of Civil Engineers.

FACAOARU I., BuCuR V., 1974. Ultrasonic methods for investigating the poplar wood anisotropy. IInd International Symposium R.I.L.E.M.. New Developments in non-destructive testing of non-metallic materials, vol. I, 66-75.

LEE I.D., 1958. A non-destructive method for measuring the elastic anisotropy of wood using an ultrasonic pulse technique. Journal of the Institute of Wood Science, 1, 43-57.

LEE I.D., 1965. Ultrasonic pulse velocity testing considered as a safety measure for timber structures. Second Symposium on the non-destructive of wood, Washington, 185-205.

Maeglin R., 1979. Increment cores, how to collect, handle and use them. Forest Products Laboratory, General technical report, F.P.L. 25.

NePVeu G., 1980. Communication personnelle sur l'interprétation de la variance.

Perrin J.R., 1980. Communication personnelle sur la programmation des données expérimentales.

Polge H., Thiercelin F., 1979. Growth stress appraisal through increment core measurements. Wood Science, 12 (2), 86-92.

Polge H., 1980. Wood quality appraisal through non-destructive testings. I.U.F.R.O., Division V, Conference Oxford, 8-16 April.

Tryon E.H., Lifwin P.J., Hamilton J.R., Renton J.J., 1976. Three methods of determining hardness of increment core segments. Wood and Fiber, 7 (4), 287-292.

Reineke L.H., DAvis C.N., 1966. A hard instrument for evaluating wood compression. Forest Product Journal, 16 (5), 15-18.

Vinh T., Chevalier Y., Le Nizerley D., 1970. Mécanique des matériaux composites. Caractérisation mécanique. Etudes dynamiques. Paris, I.S.M.C.M. 